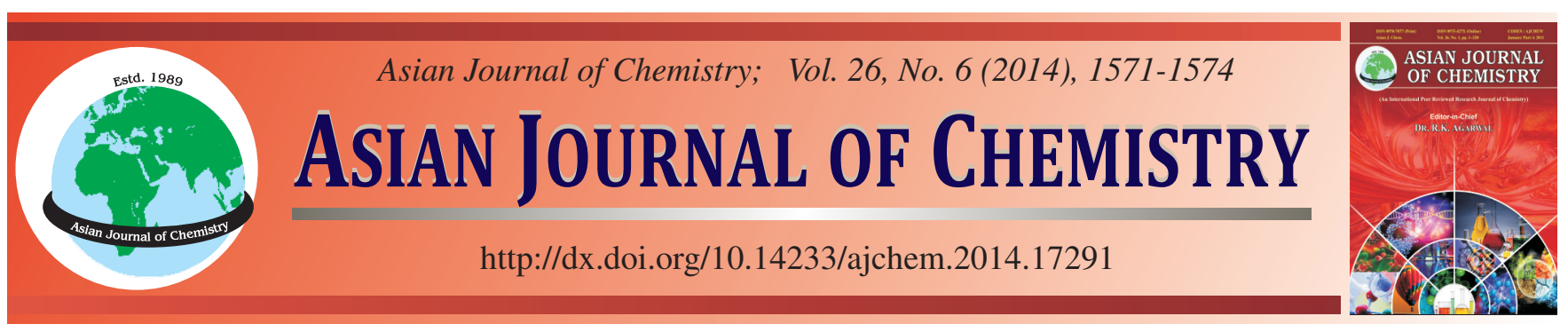

\title{
Ceramic-Based Microfluidic Device for Separation of Magnetic Particles in Continuous Flow $\dagger$
}

\section{Ji-Yun Seon, Young Joon Yoon*, Kwang Yeon Cho, Chang-Yeol Kim, Jong-Hee Kim and Hyo Tae Kim}

Nano-Convergence Intelligence Material Team, Korea Institute of Ceramic Engineering and Technology, 77, Digital-ro 10-gil, Guemcheongu, Seoul 153-801, Republic of Korea

*Corresponding author: E-mail: yjyoon@kicet.re.kr

\begin{abstract}
A ceramic-based microfluidic biochip for the application to separate the magnetic particles in the continuous flow was fabricated. To realize the various functions in a biochip, passive mixer, passive filter and active separator was integrated. Active separation was performed by external permanent magnet. A ceramic-based microfluidic chip was fabricated by LTCC (low temperature co-fired ceramic) process combined with photolithography. Through the addition of photosensitive polymer into LTCC slurry, it was possible to form a microchannel in a ceramic body by UV photolithography. To realize the magnetic separation under the applied magnetic field in a continuous flow, the separation chamber was designed to have multi-channel system with different channel width. To check the performance of a ceramicbased microfluidic device, microfluidic parameters were optimized considering both the hydrodynamic and magnetic force of the ceramicbased chip.
\end{abstract}

Keywords: Ceramic, Low temperature co-fired ceramic, Microfluidics, Separation, Magnet.

\section{INTRODUCTION}

The lap-on-a-chip (LOC) has been widely studied for the separation a specific particle in many fields such as chemical-, bio-, medical applications ${ }^{1-3}$. It has been a good advantage that there are various separation methods applicable to LOC such as hydrodynamic, magnetic, acoustic, dielectrophoretic and optical way ${ }^{4-6}$. Among these various separation methods, the magnetic separation has been received a great attention to fabricate the bio or medical of sensor using magnetic particle and external magnetic force due to its simple configuration. Nowadays, many efforts are mostly focused on micro-fabrication technology for the integrated structure in a single LOC combining the separation technology above mentioned ${ }^{6}$.

Si-based MEMS technologies can make it possible to integrate a lot of components, such as microchannel, microreactor, micropump and microvalve, on a chip. However, Sibased chip require high cost to fabricate a fully integration system ${ }^{7}$. The polymer materials, such as polymethylmethacrylate (PMMA) and polydimethylsiloxane (PDMS), makes it much easier to fabricate the individual microfluidic devices with low cost, but the problems of integration of each device on a chip are still remained ${ }^{7}$. On the other hand, considering the unique properties of ceramic material and related process, it could be a promising candidate as a substrate to integrate the individual microfluidic module because it is possible to make three dimensional integrated microstructures effectively through low temperature co-fired ceramic (LTCC) technology. Conventional LTCC technology makes it easier to integrate the components such as resistors, capacitors and inductors in the substrate $e^{8,9}$.

In this paper, a ceramic-based ceramic chip for the application to magnetic particle separation using external magnet was fabricated by LTCC technology. It was composed of two parts of mixing channel and separation channel. In a ceramicbased chip, microchannel could be formed by UV photolithography because the photosensitive components were added to prepare the slurry of LTCC. In the mixing zone, U-type channel which showed a high mixing efficiency in the passive mixer was used. To separate the polystyrene beads with magnetic film coating on the surface in a continuous flow configuration, the microchannel was designed with the unbalanced channel width for sorting particles in a specific position. Magnetic force was modulated by adding the unit of external 
magnet and its flux was optimized considering the chip design. The performance of magnetic particle separation was evaluated in a ceramic-based chip varying the flow rate of medium suspending the magnetic particles.

\section{EXPERIMENTAL}

The main ceramic material for LTCC slurry was Ca-modified amorphous cordierite and the photosensitive components was added for photolithography. The green sheets were fabricated by casting of LTCC slurry and those were laminated in a moderate thickness $1 \mathrm{~mm}$. Through the UV photolithography, microchannel could be patterned on the laminated LTCC-based sheets. Finally, it was sintered at $800{ }^{\circ} \mathrm{C}$ to obtain a rigid body. The experimental details for the fabrication procedure were described in a previous publication ${ }^{10}$.

After the sintering process, the ceramic-based chip was covered with transparent polydimethylsiloxane (PDMS) polymer (Sylgard 184, Dow Corning) for microfluidic experiments. In the mixing zone, mixing efficiency of two kinds of solutions was evaluated. One of the solutions was a $2 \mathrm{mM}$ fluorescenin (F2456; Sigma-Aldrich, St. Louis, MO) solution and another was 1 st butter using de-ionized water. For the separation experiment, fluorescenin was replaced with fluorescence superparamagnetic microspheres (Bangs Laboratories, Inc). In the separation channel zone, additional 2nd buffer solution inlet was employed to control the flow of magnetic microsphere suspended medium. The 2nd buffer solution used de-ionized water. The external magnet $(\mathrm{NdFeB})$ with the magnetic flux of 200-500 mT was used. The fluorescence images from fluorescenin solution and fluorescence microspheres were detected by a SteREO Discovery V 12 (Zeiss, Oberkochen, Germany) microscope with a MacroFire charge-coupled device (CCD) camera (Optronics, Goleta, CA).

\section{RESULTS AND DISCUSSION}

Fig. 1(a) showed the overall image of a ceramic-based chip, which was composed of mixing and separation channel zone. Main body was fabricated by conventional LTCC process and microfluidic channel in a ceramic body was formed by photolithography process, as described earlier. In the mixing zone, two inlets for injecting sample solution and 1st buffer solution were positioned. Two kinds of solution were performed a laminar flow after injection from the each inlet and those were mixed by passing through the U-type channel, which showed total length of $15 \mathrm{~cm}$ curved of five turning, width of $500 \mu \mathrm{m}$ and height of $50 \mu \mathrm{m}$. A long channel length was preferred in the passive mixer to achieve a sufficient mixing efficiency. In the separation zone, a main channel was divided into two channels, which was designed with unbalanced channel width as shown in Fig. 1(b). A total width of separation chamber was $1.2 \mathrm{~mm}$ and it was divided into wide channel (width of $600 \mu \mathrm{m}$ ) and narrow channel (width of $400 \mu \mathrm{m}$ ). Unbalanced channel width was employed to separate only the magnetic particles effectively through the manipulation of non-inertial magnetic force.

In the separation of particles in a continuous flow, the behaviour of particles is strongly influenced by the structure factor of microfluidic channel and the flow rate of solution. In

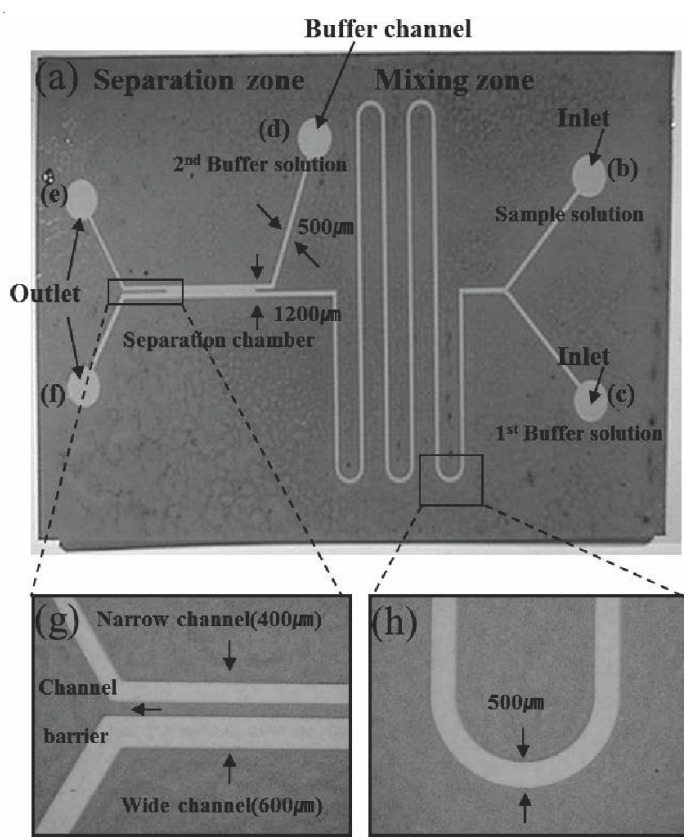

Fig. 1. (a) Optical images of passive mixing combined with magnetic separation channel Details including dimension are shown in (b) to (h)

other words, most of the particles suspended in the solution would be guided by following laminar streamlines.

Fig. 2 shows the concept of an integrated ceramic-based chip. Sample solution with target material and 1st buffer solution including magnetic particles were introduced into mixing zone. Two kinds of solutions are sufficiently mixed with each other passing through the U-type long microchannel. In the mixing procedure, it is possible to link the specific target materials with magnetic particles intentionally through the surface modification. To increase the cross-section to meet the target materials and magnetic particles, the long channel length and the high mixing efficiency of microfluidic channel are essential. In the separation zone, the additional 2 nd buffer solution was introduced with high flow rate and it made the mixed solution from the main channel to be pressed near the opposite channel wall. When the external force was not applied, the most of samples was drained out into wide channel passing through the separation chamber. At this time, when the external force by permanent magnet was applied as shown in Fig. 2, the magnetic particles suspended in the mixed solution can be selectively deflected from the main stream and those can be separated effectively into narrow channel drain.

To evaluate the passive mixing in the microchannel, the hydrodynamic flow was conducted at a low Re states and each flow rates were $0.3,1,2$ and $7.5 \mu \mathrm{L} / \mathrm{min}$. The result of mixing between fluorescence buffer and non-fluorescence buffer was analyzed by image $\mathbf{J}$ software and the mixing efficiency was summarized in Table- 1 . The mixing efficiency was maximized showing the value of $97.3 \%$ when the flow rate was $0.3 \mu \mathrm{L} /$ min. On the other hand, under the fastest flow condition at 7.5 $\mu \mathrm{L} / \mathrm{min}$, the mixing efficiency was $57.1 \%$. The mixing efficiency in the passive microfluidic mixer relied on a diffusion at the interface between two solutions. Therefore, it is better to keep the low flow rate to obtain the high mixing rate and the flow rate in the mixing zone was at $0.3 \mu \mathrm{L} / \mathrm{min}$. 


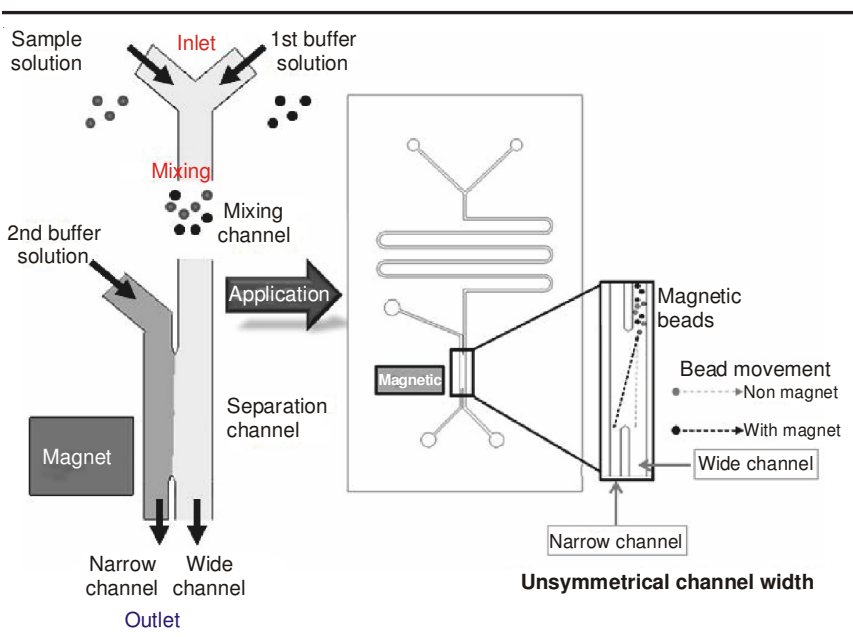

Fig. 2. Schematic diagram of a ceramic-based chip showing the concept of mixing and the separation

\begin{tabular}{|c|c|c|c|c|}
\hline \multicolumn{5}{|c|}{$\begin{array}{c}\text { TABLE-1 } \\
\text { MIXING EFFICIENCIES OF U-TYPE CHANNEL } \\
\text { PATTERN AT VARIOUS FLOW RATES }\end{array}$} \\
\hline & \multicolumn{4}{|c|}{ Flow rate of solutions $(\mu \mathrm{L} / \mathrm{min})$} \\
\hline & 0.3 & 1.0 & 2.0 & 7.5 \\
\hline Mixing efficiency (\%) & 97.3 & 94.8 & 89.8 & 57.1 \\
\hline
\end{tabular}

The streamline after mixing of both two solutions in mixing channel zone met the separation channel with the width of $1.2 \mathrm{~mm}$. Due to the different channel width between mixing channel and separation chamber, the hydrodynamic flow was subjected to a sudden expansion and dispersed into separation chamber. In this region, the 2 nd buffer solution was employed to control the flow and magnetic particles in solution can be aligned along the side wall.

Prior to the sorting of particles, we attempted to control the streamline near side wall using fluorescence solution to observe visible images. Fig. 3 showed the fluorescent solution image with the flow rate of 0.3 and $1 \mu \mathrm{L} / \mathrm{min}$ after mixing when the flow rate of the 2 nd buffer solution was fixed at $1 \mu \mathrm{L} / \mathrm{min}$. When the mixing flow rate was $0.3 \mu \mathrm{L} / \mathrm{min}$, the fluorescence

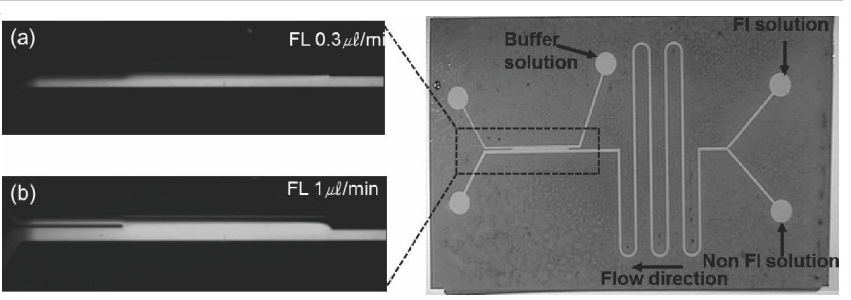

Fig. 3. Behaviour of solutions in the separation chamber with the variation of flow rate of mixed solution. The flow rate of 2 nd buffer solution was $1 \mu \mathrm{L} / \mathrm{min}$. (a) The flow rate of mixed solution of $0.3 \mu \mathrm{L} / \mathrm{min}$ and (b) The flow rate of mixed solution of $1 \mu \mathrm{L} / \mathrm{min}$

solution was drained into wide channel directly. On the other hand, when the flow rate of mixing solution was $1 \mu \mathrm{L} / \mathrm{min}$, the fluorescence solution was detected at not only wide channel but also narrow channel. Therefore, it is better to keep the flow rate of the mixed solution at $0.3 \mu \mathrm{L} / \mathrm{min}$.

To apply a ceramic-based chip to separate the magnetic particles actually, fluorescent magnetic polystyrene microsphere was introduced from the 1 st buffer inlet instead of fluorescent solution. In addition, the permanent $\mathrm{NdFeB}$ magnet was placed $0.5 \mathrm{~mm}$ from the on top of the microchip toward one side of the separation chamber, which poisoned with $2 / 3$ of whole separation chamber from outlet.

Fig. 4(a) showed the movement of magnetic beads when the magnetic force was not applied. In this case, all magnetic beads were drained into wide channel regardless of the flow rate of the 2 nd buffer solution. In the Fig. 4(a), when the 2nd buffer flow rate were increased, the magnetic bead was displaced close to the outer wall of wide channel. Fig. 4(b) showed the behaviour of the magnetic beads when the magnetic field was applied by permanent magnet. When the 2 nd buffer flows rates were 1, 2 and $4 \mu \mathrm{L} / \mathrm{min}$, the motion of magnetic beads were deflected into narrow channel toward the strong magnet field. However, it was observed that the magnetic force was weaker than the hydrodynamic force when the flow rate of the 2 nd buffer solution was over $6 \mu \mathrm{L} / \mathrm{min}$. When the perpendicular force induced by magnetic field was lower than hydro-

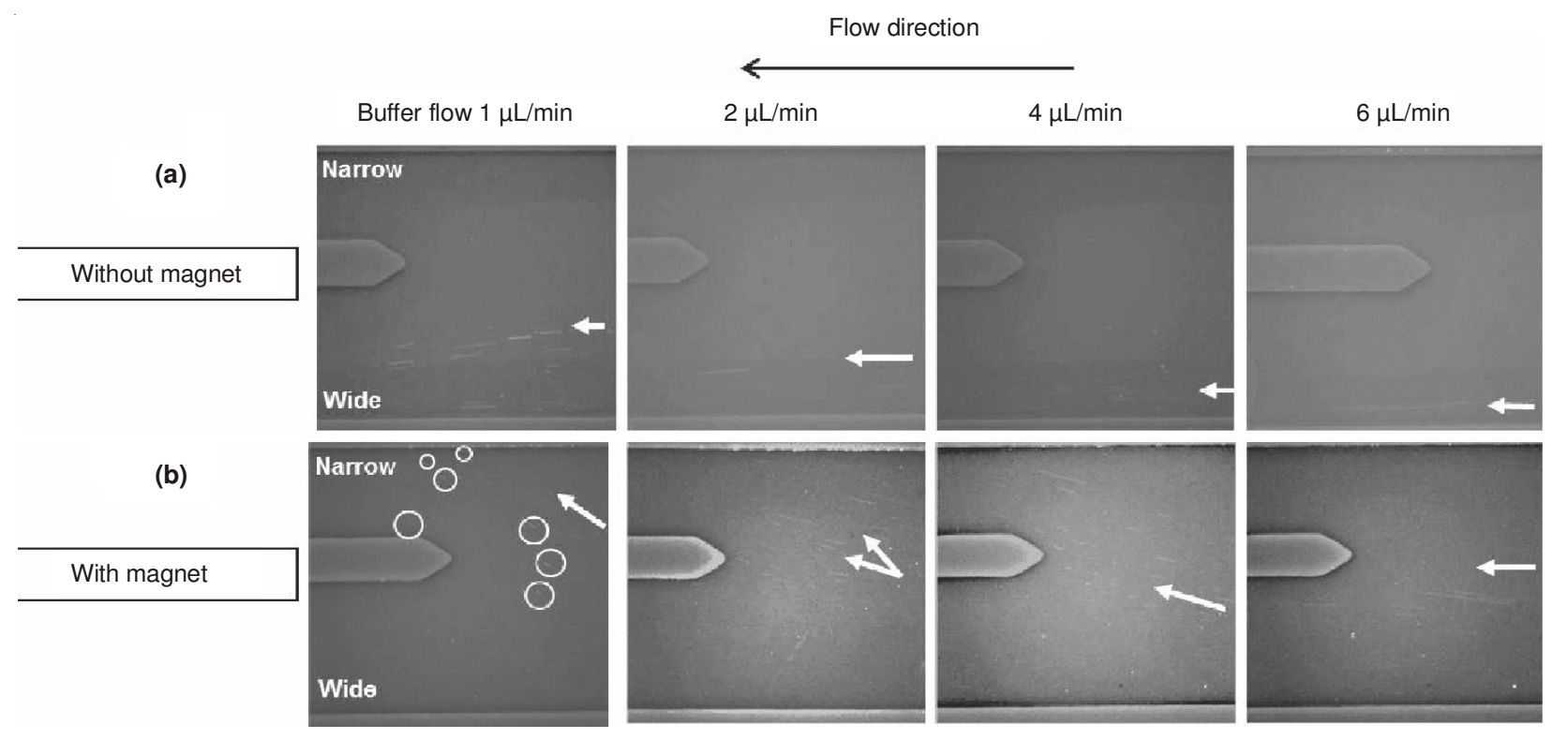

Fig. 4. Behaviour of magnetic polystyrene microsphere in the separation chamber with the variation of the 2nd buffer solution flow rate. (a) Without the magnetic field and (b) with the magnetic field 
dynamic flow, the movement of magnetic beads suspended in the solution less shifted into upper trajectory relatively. In other words, the degree of deflection of the magnetic particles can be calculated by the sum of two factors. One is the vector for the magnetically induced flow velocity on the particles and another is the vector for the velocity of the hydrodynamic flow in the microfluidic channel. It was confirmed that the separation of magnetic particles in a ceramic-based chip was well controlled by the modulation of both magnetic and hydrodynamic force.

\section{Conclusion}

A ceramic-based chip for hydrodynamic mixing and magnetic separation was fabricated by using LTCC process and photolithography. It was possible to separate the magnetic particles efficiently controlling both magnetic and hydrodynamic force. In the ceramic-based chip, the motion of magnetic beads were deflected into narrow channel toward the strong magnet field, when the 2 nd buffer flows rates were below $4 \mu \mathrm{L} /$ min. A LTCC process would be a promising technology for embedding the complex and various microfluidic components in a chip due to its unique nature for easy $3 \mathrm{D}$ integration.

\section{ACKNOWLEDGEMENTS}

This work was supported from the R \& D program by Korea Institute of Ceramic Engineering and Technology.

\section{REFERENCES}

1. A. Lenshof and T. Laurell, Chem. Soc. Rev., 39, 1203 (2010).

2. N. Pamme and A. Manz, Anal. Chem., 76, 7250 (2004).

3. C. Derec, C. Wilhelm, J. Servais and J.-C. Bacri, Microfluid. Nanofluid., 8, 123 (2010).

4. Z. Zhu, J.J. Lu and S. Liu, Anal. Chim. Acta, 709, 21 (2012).

5. P.-A. Auroux, D. Iossifidis, D.R. Reyes and A. Manz, Anal. Chem., 74, 2637 (2002).

6. N. Pamme, Lab Chip, 7, 1644 (2007)

7. P. Abgrall and A.-M. Gue, J. Micromech. Microeng., 17, R15 (2007).

8. L.J. Golonka, Bull. Polish Acad. Tech. Sci., 54, 221 (2006).

9. K. Malecha and L.J. Golonka, Microelectron. Reliab., 48, 866 (2008).

10. J. Choi, Y.J. Yoon, Y.-S. Choi, H.T. Kim, J. Kim, J.-H. Lee and J.-h. Kim, J. Ceramic Proc. Res., 12, 146 (2011). 